\title{
Functional maturity of tricuspid and mitral valves in school children evaluated by echocardiography
}

\author{
H Sugiyama, M Hoshiai, T Tan, S Nakazawa
}

Heart 2005;91:1479-1480. doi: 10.1136/hrt.2004.057083

$\mathrm{T}$ he prevalence of valve regurgitation in healthy people has been previously examined by echocardiography. ${ }^{1-4}$ Pulmonary regurgitation or tricuspid regurgitation (TR) was common, ${ }^{1-4}$ mitral regurgitation (MR) was less common, and aortic regurgitation was generally undetectable. ${ }^{2-4}$ In addition, the prevalence of regurgitant signals varies with age or sex. Assuming that a low prevalence of regurgitant signals indicates functional maturity, examining changes in the prevalence and severity of these signals is useful for evaluating the functional development of the valves. In this study, we examined the prevalence and severity of TR and MR with colour Doppler echocardiography in school children and healthy adults.

\section{METHODS}

The committee for research in human subjects of the University of Yamanashi approved the protocol. We enrolled 253 children (84 junior school children with a mean (SD) age of $9.8(0.3)$ years and 169 junior high school children aged 13.0 (0.3) years) and 45 healthy adult volunteers aged 38.5 (7.4) years in this study. All participants underwent ECG and a physical examination before the study. No findings were abnormal.

The colour Doppler examination was performed with an echocardiographic system (Sonos 2500; Hewlett Packard, Andover, Massachusetts, USA) with a $3.5 \mathrm{MHz}$ transducer. All participants were continuously examined by a single skilled examiner over two days. Data were recorded on videotape and evaluated by two independent investigators who were blinded to the study design. Regurgitation was examined in the parasternal long axis and apical four chamber views. The Doppler colour gain was optimised by conventional methods. The severity of regurgitation was semiquantitatively assessed on a five grade scale by determining the intrusion distance of the regurgitant signal from the tricuspid or mitral valve orifice in the apical four chamber view as follows: none (no signal); trivial (small amount of signal); mild (less than one third of the atrium); moderate (from one to two thirds of the atrium); and severe (more than two thirds of the atrium).

All data are presented as mean (SD). Data were analysed by the Spearman rank correlation, the Kruskal-Wallis test, and the Mann-Whitney $U$ test. A probability value of $\mathrm{p}<0.05$ was considered to be significant.

\section{RESULTS}

TR and MR were detected in $88 \%$ and $26 \%$, respectively, of school children. In the tricuspid valve, most regurgitations were classed as trivial. By contrast, in the mitral valve no signal was generally observed. Then, the prevalence decreased with severity. The overall severity of TR was significantly greater than that of MR $(\mathrm{p}<0.001)$.

TR was more severe in junior school children than in junior high school children ( $p<0.001$; fig 1A) and more severe in boys than in girls $(\mathrm{p}<0.05$; fig $1 \mathrm{~B})$. By contrast, MR did not differ significantly between age groups or between sexes. The severity of TR was significantly correlated to that of MR in school children $(\mathrm{p}<0.001)$.

TR and MR were detected in $51 \%$ and $13 \%$, respectively, of adults. In addition, TR was significantly more severe than MR $(p<0.001)$. Furthermore, the severity of TR was correlated to that of MR $(\mathrm{p}<0.001)$. TR and MR did not differ significantly between sexes.

TR was significantly more severe and more common in school children than in adults $(\mathrm{p}<0.001$; fig 1$)$.

\section{DISCUSSION}

The prevalence of atrioventricular valve regurgitation varied from $2-45 \%$ in $\mathrm{MR}$ and from $6-78 \%$ in TR in previous reports. ${ }^{1-4}$ The large variation in these results is probably due
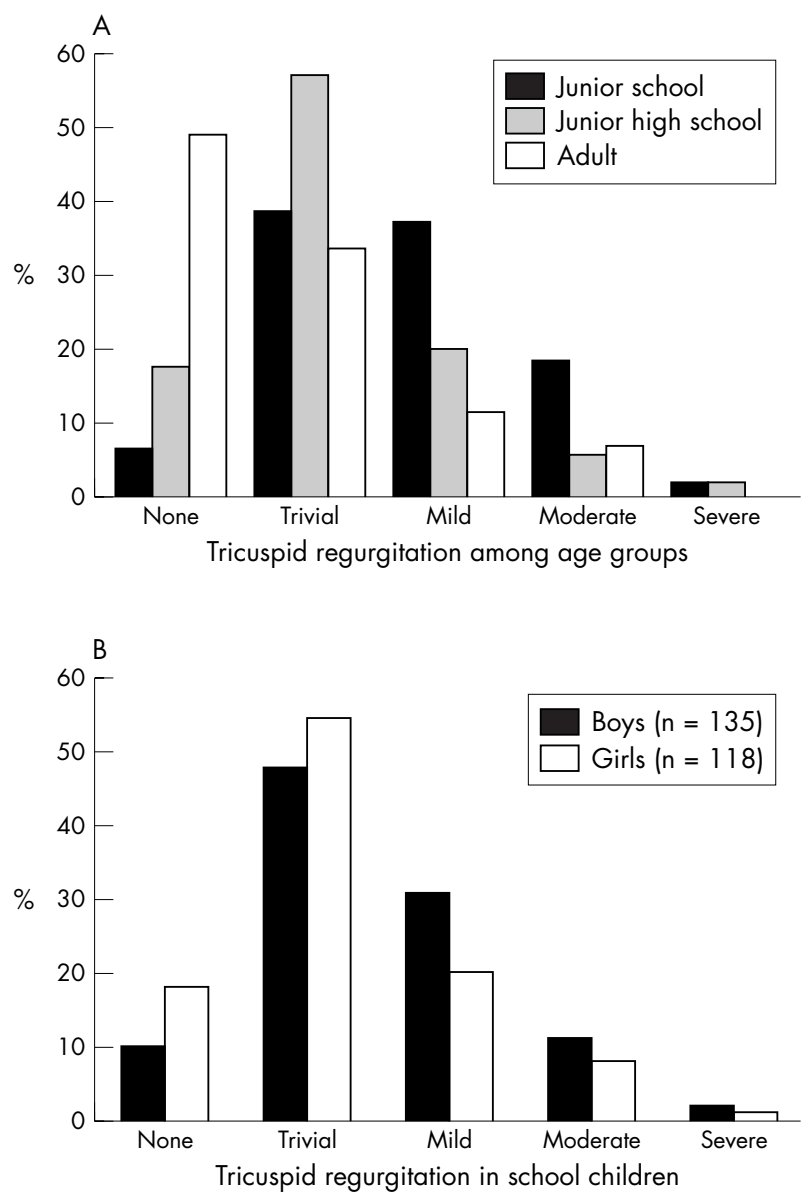

Figure 1 (A) Comparison of the prevalence and severity of tricuspid regurgitation between school children and adults. Prevalence and severity decreased with age $(p<0.001)$. (B) Comparison of the prevalence and severity of tricuspid regurgitation between school boys and girls. Prevalence and severity of tricuspid regurgitation were greater in boys than in girls $(p<0.05)$. 
to differences in the methods and criteria. In the present study, the severity of the regurgitant signal was semiquantitatively assessed on a five grade scale. In addition, to minimise the intraobserver and interobserver variabilities, the same skilled examiner continuously recorded the echocardiograms of all participants over two days.

In the present study, TR and MR were detected in $88 \%$ and $26 \%$, respectively, of school children. When apparent regurgitation was defined as greater than trivial, these prevalences were $36 \%$ and $5 \%$, respectively.

The initial structure of the tricuspid valve leaflets is more complicated than that of the mitral valve leaflets. Moreover, the morphology of the mitral valve orifice is ellipsoid, whereas that of the tricuspid valve orifice is distorted. On the basis of these factors, the tricuspid valve is considered to be more vulnerable to regurgitation. In the present study, the prevalence and severity of TR decreased with age. Furthermore, TR was more common and more severe in men than in women. This suggests that the tricuspid valve matures functionally earlier in women than in men during adolescence.

The trends in the prevalence and severity of MR differed from those of TR. However, the prevalence and severity of TR were associated with those of MR. One possible explanation for this relation is that the geometrical change of one atrioventricular valvar annulus may affect that of the other. Another possibility is that connective tissue consisting of atrioventricular valves may not be mature enough to support the valve structure.

In adults, similar to school children, TR was more common and more severe than MR. However, TR was less common and less severe in adults than in school children. These results suggest that the tricuspid valve matured over time. On the other hand, the mitral valve functionally matured at a younger age. The atrioventricular valve has been reported to deteriorate with age due to tissue degeneration in elderly people. ${ }^{5}$ We therefore suggest that the atrioventricular valve tissue becomes more durable and matures during adolescence but begins to degenerate in older age.

All regurgitation signals were considered to be functional. No study participants had enlarged atria or abnormal valve structures.

The present study showed a significant difference between the functional maturity of the tricuspid and mitral valves: the mitral valve is morphologically robust towards regurgitation in childhood, whereas the tricuspid valve takes decades to mature functionally. Therefore, the prevalence and severity of both TR and MR with respect to aging should be considered.

\section{Authors' affiliations}

H Sugiyama, M Hoshiai, T Tan, S Nakazawa, Department of Paediatrics, Faculty of Medicine, University of Yamanashi, Yamanashi, Japan

Correspondence to: Dr Hisashi Sugiyama, Department of Paediatrics, Faculty of Medicine, University of Yamanashi, 1.110 Shimokato, Tamaho, Yamanashi, 409-3898, Japan; sugiyama_hisashi@hotmail. com

Accepted 10 February 2005

\section{REFERENCES}

1 Van Dijk AP, Van Oort AM, Daniels O. Right-sided valvular regurgitation in normal children determined by combined colour-coded and continuous-wave Doppler echocardiography. Acta Paediatr 1994;83:200-3.

2 Akagi $\mathrm{T}$, Inove $\mathrm{O}$, Ohara $\mathrm{N}$, et al. Valvular regurgitation in patients with Kawasaki disease and in healthy children: a pulsed Doppler echocardiographic study. J Cardiol 1989;19:787-96.

3 Brand A, Dollberg S, Keren A. The prevalence of valvular regurgitation in children with structurally normal hearts: a color Doppler echocardiographic study. Am Heart J 1992; 123:177-80.

4 Yoshida K, Yoshikawa J, Shakudo M, et al. Color Doppler evaluation of valvular regurgitation in normal subjects. Circulation 1988;78:840-7.

5 Aronow WS. The older man's heart and heart disease. Med Clin North Am 1999;83:1291-303.

\section{FROM BMJ JOURNALS}

\section{Milk consumption, stroke, and heart attack risk: evidence from the Caerphilly cohort of older men}

P C Elwood, J J Strain, Paula J Robson, Ann M Fehily, Janie Hughes, Janet Pickering, Andy Ness

Please visit the Heart website [www.heartinl. com] for a link to the full text of this article.
Objective: To examine associations between milk consumption and incident heart disease and stroke.

Design: A representative population sample of men was asked to weigh and record their food intake for seven days. The total consumption of milk was obtained from these records. Details of all deaths and vascular events were collected during the following 20 years. Incident ischaemic strokes and heart disease events were diagnosed by standard criteria.

Setting: The Caerphilly cohort, a representative population sample of men in South Wales, aged 45-59 when first seen in 1979-83.

Participants: A representative 3:10 subsample of the men in the cohort.

Main results: 65 men ( $87 \%$ of those approached) returned satisfactory seven day diet diaries. After adjustment, the relative odds of an event in the men whose milk consumption was the median or higher, relative to those with lower intakes of milk, were $0.52(0.27$ to 0.99$)$ for an ischaemic stroke and 0.88 (0.56 to 1.40) for an ischaemic heart disease event. Deaths from all causes were similar in the two milk consumption groups (relative odds 1.08; 0.74 to $1.58)$.

Conclusions: These results give no convincing evidence of an increased risk of vascular disease from milk drinking. Rather, the subjects who drank more than the median amount of milk had a reduced risk of an ischaemic stroke, and possibly a reduced risk of an ischaemic heart disease event. These conclusions are in agreement with the results of a previously reported overview of 10 large, long term cohort studies based on food frequency intake records.

A Journal of Epidemiology and Community Health 2005;59:502-505 